\title{
A Framework to Support Creativity in Multimedia Information Design
}

\author{
Kumiyo Nakakoji ${ }^{1,4}$, Kouhei Suzuki $^{2}$, Nobuo Ohkura ${ }^{3}$, Atsushi Aoki ${ }^{1}$ \\ ${ }^{1}$ Software Engineering Laboratory, Software Research Associates, Inc., \\ 3-12, Yotsuya, Shinjyuku, Tokyo, JAPAN \\ ${ }^{2}$ Information Technology Promotion Agency, MITI, Tokyo, JAPAN \\ ${ }^{3}$ PFU Inc., Tokyo, JAPAN \\ ${ }^{4}$ Cognitive Science Laboratory, Graduate School of Information Science, \\ Nara Institute of Science and Technology, JAPAN \\ kumiyo@sra.co.jp,k-suzuki@ipastc.stc.ipa.go.jp, ohkura@pfu.co.jp, \\ aoki@sra.co.jp
}

\begin{abstract}
Creating good multimedia information design requires a variety of domain knowledge, including knowledge about color, sound, movies and multimodal effects. Our eMMa (Environment for MultiMedia Authoring) project has focused on designing, building and assessing a computational system that supports casual users in designing multimedia information, such as creation of world-wide-web home pages. Exercising creativity in such design tasks is to find a "right" balance between innovativeness and usefulness of designed artifacts. This paper presents a framework of eMMa to support creativity in the both dimensions, with a brief scenario to illustrate how the eMMa system is used. We discuss case studies we have conducted in several academic and industrial sites, which demonstrates that users showed some creative aspects in their multimedia information design.
\end{abstract}

\section{KEYWORDS multimedia information design, creativity, knowledge-based support \\ 1. MULTIMEDIA INFORMATION DESIGN \\ Multimedia information design requires a wide range of design knowledge. Designers need to know how people perceive representations of text, color, sound, visual images and movies, as well as their psychological and cultural associations. In addition, there are some studies that specifically focus on the effectiveness of \\ multimedia information design (Bearne, Jones, Sapsford-Francis1994, Tufte, 1983, Faraday, Sutcliff, 1997). \\ A well-designed multimedia information communicates its underlying intention to readers (or audience) as precisely, as concisely, and as rapidly as possible. On the other hand, poorly designed multimedia information, for example, arbitrary colored home pages on world-wide-web, is not only aesthetically unpleasant but also misleading. It may}

Human-Computer Interaction: INTERACT'97 S. Howard, J. Hammond \& G. Lindgaard (editors)

Published by Chapman \& Hall OIFIP 1997 
unfocus the point of the message, being unable to "correctly" communicate its intention to readers.

While many textbooks and courses are available for studying about each media, it is impossible to expect casual users to attend to such a wide range of information resources. By casual users, we mean those who are not professional multimedia title designers but those who use multiple media as a means to communicate their intention in their everyday practice. On-line manual for software products of a small company, personal recipe for home cooking, and a World-wide-web homepage for a local restaurant are example of such casual uses of multimedia information.

To support multimedia information designers in casual occasions (as opposed to support for professional multimedia title designers), we have developed a knowledge-based authoring support system, eMMa (Environment for MultiMedia Authoring) (Nakakoji et al. 1995, Nakakoji, Aoki, Reeves, 1996). Based on a human-computer collaborative problem solving model (Terveen 1995), eMMa supports users in selection of colors in their creation of visual images and search of a library of hundreds of reusable images. Experiences and "know-how's" are encoded as rules in eMMa's community knowledge-base, and used to map user's high-level requirements (e.g., to create a home page for young consumers) to detailed design decisions (e.g., which color to use as a background).

One goal in supporting users in multimedia information design, or in other design domains, is to build a computational support in such a way as to enhance users' creativity rather than imposing users of a single "right way to design." While the meaning of the term creativity may vary widely, we use the term to mean to bring existing design concepts into unseen and even unthought, yet valuable ways of usage (McLaughlin, Gero 1989). A multimedia information design is creative if synthesis of concepts and media usage is innovative and at the same time useful.

eMMa amplifies users' creativity by automatically volunteering information relevant to the users' current design situation (Fischer, Nakakoji, 1994). The system makes suggestions for users, which the users may have never thought of before, help them become aware of new aspects for their design tasks, leading to innovative artifacts. The system criticizes users' partial designs for improvement using its knowledge-base, leading to useful artifacts. In addition, the system's knowledgebase is shared and evolved by a community of users. Thus, their creative design can be supported in the social context (Csikszentmihalyi, Sawyer, 1995). By integrating the two dimensions of creativity with respect to social factors, the system supports people to produce creative multimedia information design.

We have conducted case studies by asking ten subjects to use eMMa to assess our approach. They are from both industry and academia, ranging from industrial design, architectural design, software engineering, web-page design, artificial intelligence, and cognitive science. We asked them to perform seven tasks to create visual images (e.g., design of background for a certain purpose) followed by interviews. We observed that based on the subjects' background, their approaches toward the tasks are quite different. In any cases, however, eMMa's knowledge-based support helped them produce artifacts in unpredicted (therefore psychologically innovative) and valuable ways.

In what follows, we first briefly describe how eMMa is used to create multimedia information design illustrated with a scenario. This scenario is drawn from a real task-flow conducted by one of the subjects in our case studies. Then, we outline a framework for computer-supported creativity. We describe theories behind our approach, and system requirements based on the framework. Next, we discuss our case studies to demonstrate how eMMa supports creativity based on the framework.

\section{THE AMMa SYSTEM}

eMMa (Environment for MultiMedia Authoring) (Nakakoji et al. 1995, Nakakoji, Aoki, Reeves, 1996) is a prototype system built on VisualWorks Smalltalk 2.5, which runs on PCs, Macintoshes, and Sun Workstations, to support casual users in producing good multimedia information designs. The system is designed for a single user, but its knowledge-base can be shared and evolved by multiple users, thereby supporting asynchronous collaboration.

\subsection{Overview of eMMa}

eMMa helps users in a collaborative manner (Terveen 1995). That is, users of the system make design 
decisions, and create and evolve design artifacts. The system monitors users' design tasks, and uses its knowledge-base to make suggestions for improvement or to identify potentially problematic situations in the users' current design. Contents of the knowledge-base can be modified by users as the system is used.

With the current implementation, the system helps users in three ways. First, the system helps users decide which colors to use in which combinations and in what proportions, based on the Munsell Color Theory (Sidelinger 1985). This functionality is called eMMaC - eMMa's Color-critiquing component (Nakakoji et al. 1995). Users specify a region anywhere on the screen, and asks eMMaC to criticize the balance of strength of each color used in the specified region with respect to each area. eMMa computes the balance by quantifying colors for each pixel of a specified image region using the Munsell Color system. Detailed descriptions of eMMaC are provided in (Nakakoji et al. 1995).

Second, the system helps users search for reusable visual images from a library of hundreds of GIF images. eMMa's ImageSearcher component (Aoki et al. 1995) allows users to formulate a query using image properties, such as color luminance, the center of gravity in terms of darkness of the image, colors used in specific areas of the image, free-hand border sketch and another image to retrieve similar images. ImageSearcher uses the information specified in the query to rank-order images of a set of GIF images by the degree of "match." Aoki et al. (1995) provides detailed descriptions of its matching algorithms.

Third, the eMMa-SPEC component (Nakakoji, Aoki, Reeves 1996) allows users to specify abstract requirements for their information design task, such as an intended type of audience, atmosphere to bring in, or a purpose. The system then, makes suggestions about color usage or other design decisions based on the specified requirements. Such inference is made through rules stored in its knowledge-base. Users can browse the rules and add or modify rules if desired.

In what follows, we present a scenario illustrating how to use eMMa in designing multimedia information. The scenario is based on an actual task-flow performed by one of the subjects we used in our case studies. Detailed discussions of eMMa's implementation can be found in somewhere else (Nakakoji et al. 1995, Nakakoji, Aoki, Reeves 1996, Aoki et al. 1995). Section
4 presents more detailed discussions about the case studies.

\subsection{A Scenario of Using eMMa}

A subject is given a task to design the background for a given image of a chair (see Figure 1). The goal is to design a poster for an industrial exhibition to demonstrate the chair.

Given the requirements, the subject first specified conditions for the task using eMMa-SPEC (Figure 2). By considering that the image is for an industrial exhibition, he thought that most of attendees for the exhibition would be engineers in their forties. He wanted to make the image precise, with a purpose of marketing in his mind.

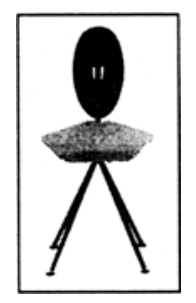

Figure 1: A given task: to decide background for industrial exhibition

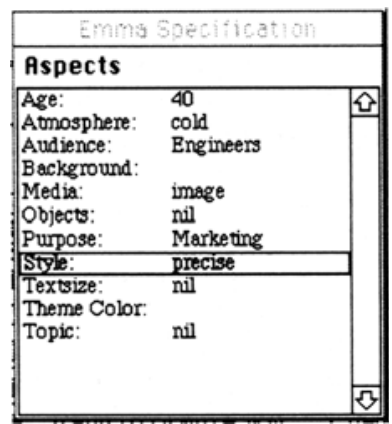

Figure 2: eMMa-SPEC

When the subject finished choosing aspects for his task using eMMa-SPEC, eMMa presented him a list of suggestions to use purple, blue, and green (see Figure 3). Those suggestions were associated with eMMaĺs rules (see the middle window in Figure 3). The system 
described why those suggestions were made; for example, suppose he wondered why blue is suggested. The bottom window in Figure 3 described that it is because he specified that intended audience would be engineers and blue brings in seriousness, which engineers would prefer.

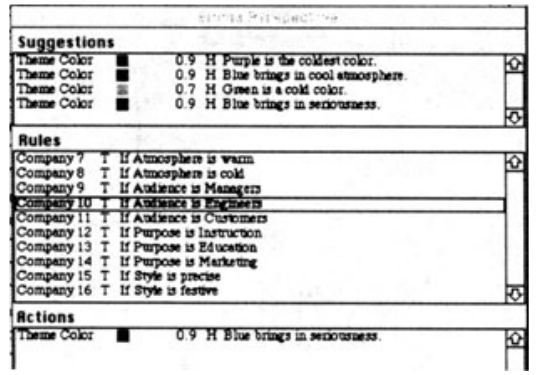

Figure 3: Suggestions made by eMMa

By looking at the suggestion, he decided to use something blue. Additionally, terms that appeared in the suggestions, such as seriousness and cold, reminded him of something "engineer-ish." $\mathrm{He}$ then remembered that there are images available in eMMa that he could use as background. So, he decided to search for pictures with straight lines. Using eMMaĺs ImageSearcher, he drew a sketch consisting of several orthogonal lines (see Figure 4). He also specified that he was interested in blue (not shown).

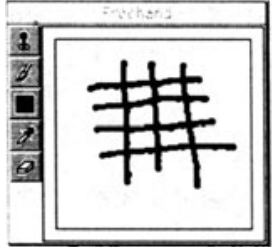

Figure 4: Free-hand sketch produced to retrieve "engineering-ish" photo images

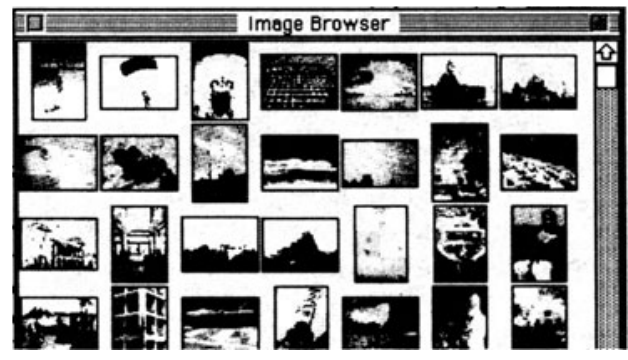

Figure 5: Ordered photo images based on the subject's query (see Figure 4)

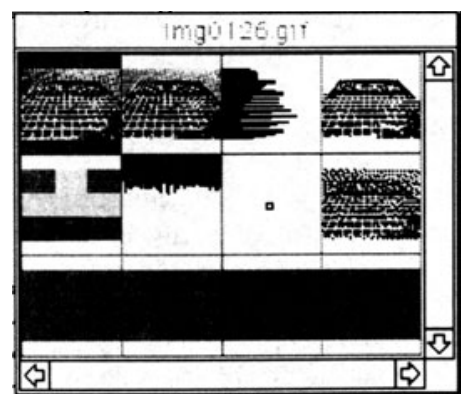

Figure 6: Automatically identified properties of an image

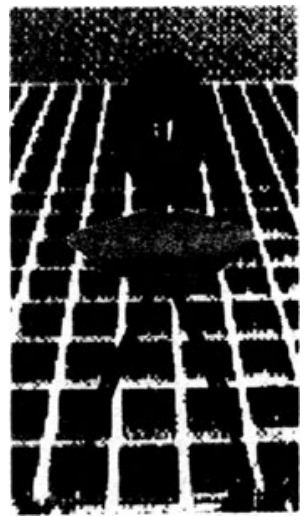

Figure 7: The subject's final design 
Based on his queries, ImageSearcher ordered pictures according to the degree of match to the query (Figure 5). eMMa used automatically identified properties of each image to compute the degree of match (Figure 6 illustrates a set of identified properties from the building image). He found a nice picture of a building with blue sky, and decided to use it as background for the given task. Figure 7 illustrates his final design.

\section{CREATIVITY}

\subsection{A Model for Creativity}

While creativity is often associated with art, we are concerned with creativity that is required in everyday work practice. This type of creativity is in most cases not historical, but psychological (Boden, 1991). A resulting product is not necessarily novel or original to a community but is personally or psychologically novel to the individual who produced it.

While professional multimedia title authors may demonstrate historical creativity in their media usage, creativity in designing multimedia information in casual situations, such as a design of personal home pages in the World-Wide-Web, is in most cases, psychological. Owners of homepages, for example, demonstrate their own creativity using unfamiliar colors and fancy graphics in their background, and attaching sound to their homepages by looking at other people's home pages.

Creative artifacts needs to be both innovative and valuable. The product must be novel so that it is not a part of existing well-known solution. On the other hand, if the product is not useful, or of little value, it cannot be regarded as creative.

Our approach to use computer systems to support creativity is to integrate the two dimensions (see Figure 8). The system helps people become aware of new ideas by presenting information relevant to the people's current problem situation. Since the relevance is measured based on the system's domain knowledge, the system's volunteered information sometimes surprises users. It is left to the users to judge the usefulness of this volunteered information.

The system helps people improve their designed artifacts. The system has design knowledge and can identify problematic situations in the users' partial designs in the form of critiquing messages (Fischer, et al. 1993, Nakakoji, Sumner, Harstad 1994). Our previous work has identified that it is important to criticize the artifact at the "right" time, and to provide rationale behind the criticisms (Fischer et al. 1993).

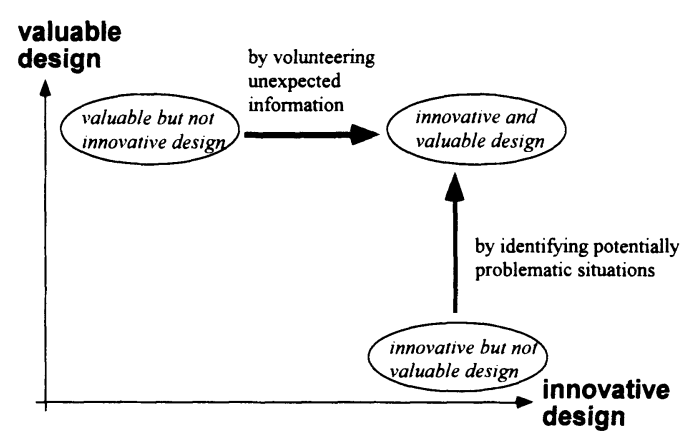

Figure 8: Two dimensions of creativity and their system support

Csikszentmihalyi and Sawyer (1995) argue that although the moment of creative insight usually occurs in isolation, it is surrounded and contextualized within an ongoing experience that is fundamentally social. They have developed a model that illustrates creative processes in social context. A creative process consists of four steps: preparation, incubation, insight, and evaluation. In the preparation phase, people identify problems, goals and constraints for achieving a task. In the incubation phase, people filter information from conscious awareness to the subconscious to be used for creative insight. In the insight stage, people actually produce something creative, finding new associations among existing objects. Finally, in the evaluation phase, people assess whether their insight is valuable.

Csikszentmihalyi and Sawyer stress the importance of embedding the creative process in social context, and the preparation, incubation, and evaluation processes use input from the community. That is, people use outside information to prepare, acquire potentially useful information from the community, and when they come up with creative insight by themselves, they use information from outside to evaluate their insight. 


\subsection{Creativity Supported by eMMa}

As we discussed above, the preparation and incubation phases help people produce innovative ideas, and the evaluation phase assures that their products are useful.

Figure 9 illustrates how our eMMa system supports creativity based on this model.

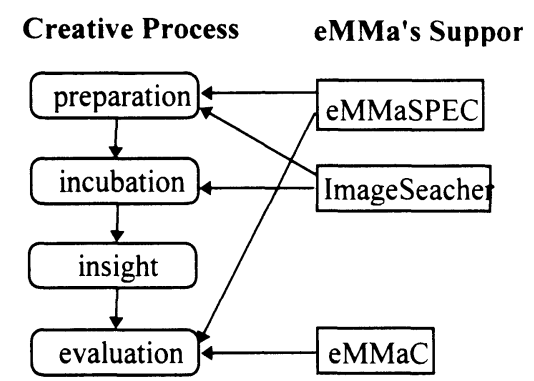

Figure 9: A Model for Creativity Support by eMMa

As illustrated in the scenario presented in Section 2.2, the system volunteers information for users relevant to the task at hand (Fischer, Nakakoji, 1994). In the scenario, the system made suggestions for the subject to use blue if he was designing for engineers as audience. This information was helpful for the subject to improve his design for the purpose as he had no idea how to map the audience-engineering requirement to a concrete design decision. Terms appearing in the suggestions, such as "seriousness" also helped the subject during the incubation phase as he gathered information relevant to his task. These rules are accumulated through the use of other users - users added rules as they used the eMMa system. Therefore, the subject indirectly communicated with a community of users through the eMMa's knowledge-base.

eMMa also helped the subject to produce an innovative artifact. The subject did not have a concrete idea for the background until he saw the picture of a building (see Figure 6). His query did not exactly state "please find a picture of a building." Instead, he drafted a sketch representing something "engineering-ish" using several orthogonal lines. $\mathrm{He}$ came up with a picture of a building, which he liked, and used it as background for his task.

\section{Case Studies}

As we designed and built the eMMa system to support casual users in multimedia information design, a concern has emerged that users of eMMa may just follow eMMaÍs suggestions and end up with the same final product. Our hypothesis was that as eMMa is built based around the model for creativity described above, products should be diverse with the help of eMMa as opposed to converging into a single outcome.

To test our hypothesis, we have conducted case studies of eMMa. We asked ten subjects to perform seven tasks using eMMa. The tasks are to design background and foreground colors and frames for given outlines and images of objects. The task presented in Section 2.1 was one of the task. Table 1 illustrates the distribution of subjects' background.

Table 1: Distribution of Subjects' Background

\begin{tabular}{|l|l|}
\hline Architecture & 1 \\
\hline Industrial Design & 1 \\
\hline Artificial Intelligence & 2 \\
\hline Web Homepage Design & 1 \\
\hline Software Engineering & 2 \\
\hline Cognitive Science & 3 \\
\hline
\end{tabular}

We prepared task descriptions on our web-site, and asked them to perform tasks at their local sites. Then, they are asked to describe what they have done through post-task interviews. Final and intermediate products are collected.

Rather than describing details of each study in detail, this paper focuses on the task presented above - to design background for a chair for industrial exhibition.

In short, no subjects produced exactly the same results. Variation of their outcomes is quite phenomenal. One used pictures as background, two subjects produced gradations of color using other drawing tools and used them as background, the others simply colored the background.

There were a variety of usage patterns of eMMa among different subjects. Two subjects simply used eMMa like a spelling checker - they designed background and asked eMMaC to criticize the color balance. Some used ImageSearcher extensively to 
find interesting usage patterns of background. One subject used a retrieved image as background. Three subjects used eMMa-SPEC to decide a main color based on the suggestions made by the system.

We have found three distinctive patterns of design processes using eMMa by subjects who have three different background: an artificial intelligence researcher, a web-homepage designer, and a software engineer. We briefly characterize each pattern and discuss how their creativity is supported by eMMa.

- Case 1: an artificial intelligence researcher

His case was basically the same as illustrated in the scenario 2.1. As discussed in Section 3, he effectively used eMMa to produce creative artifacts.

- Case 2: a web-homepage designer

She used eMMa-SPEC to characterize the task. She specified almost the same conditions as in Case-1. Once she was told by eMMa that blue should be used as a suggestion, however, she started exploring different variations of blue. She used $\mathrm{eMMaC}$ to find the right balance between the yellow of the chair and a variety of blues. She used another drawing tool to produce gradations of blues and used one of them as background for her final design. She browsed the image library, but did not do anything with it.

During the interview, she told us that it was helpful for her to be instructed to use blue by eMMa. Once being told, however, she wanted to explore for herself possible design spaces based on blue. It was relieving for her that she could use eMMaC to make sure her design decision "is good enough for the system's knowledge (originally transcribed in Japanese)."

- Case 3: a software engineer

A software engineer started trying to fill conditions using eMMa-SPEC. He mapped the industrial exhibition requirement to "audience: customer" and "style: festive" and "atmosphere: cheerful." The system, then, suggested to use warm colors to be cheerful, thereby to use yellow. He colored background to the chair yellow and asked eMMaC to adjust the balance between two yellows. The system suggested several variations of lighter yellows to use instead of his original yellow background. $\mathrm{He}$ agreed with the system, and finished his design with light yellow background.

It was interesting that this subject specified eMMaSPEC in a totally different way from the previous two cases. The concept "for industrial exhibition" was mapped to something festive and cheerful, as opposed to something "engineering-ish" as illustrated in Case-1. Based on this different mapping, the system made completely different suggestions for color: yellow, a warm color, not blue. Subsequent design processes were different from those of Case- 1 because of this different suggestion.

Through his interview, he also appreciated that the system suggested to use yellow, as he "had no idea what to do, and completely agreed with the system's suggestion." $\mathrm{He}$ also liked that eMMaC adjusted background and foreground yellows to assure him that his product is "okay." He was aware of the existence of ImageSearcher, but "never thought of using it because he found no need to find anything."

\section{DISCUSSION}

The above three cases we found in our case studies have demonstrated variations of usefulness of eMMa. While assigned tasks are quite simple (e.g., to design background), no final products were even similar to each other. This assured us that using eMMa will not impose users of a single outcome. In fact, subjects demonstrated quite a variation in their design decision making processes.

For example, the Case- 3 subject mapped the requirements to concrete conditions very uniquely. The phrase "industrial exhibition" did not remind him of engineering, but more of festive events. Based on his understanding of the problem space, the system retrieved information relevant to his framed problem (i.e., suggested to use yellow). eMMa thus helped him increase usefulness of his design and did not prevent him from exploring new problem spaces.

Case-2 subject enjoyed exploring the solution space based on the system's suggestion - to use blue. As she finished her design, she wanted to create a rule to state that "if a purpose is for industrial exhibition, you should use not blue in general but this particular blue (that she used)," but did not dare to create one. The interview 
with her revealed that the rule occurred to her as she was performing her task. This type of "creative insights" should be able to stored in the system easily. While eMMa currently requires users to directly edit Smalltalk-style predicates to add and modify such rules, an end-user modifiable rule interface needs to be built.

As future considerations for the system, many subjects wanted to have smoother integration of the components of eMMa. Seven subjects first specified their requirements, looked at the suggestions made by the system, and used the suggestion as a query to search images in ImageSearcher. Retrieved images were then evaluated using eMMaC. If this sequence of actions is automated, however, there would be no room for users' subjective interpretations. For example, Case-1 subject mapped the concept "engineering" to several orthogonal lines. We need more studies to explore the role of creativity in these mapping processes.

\section{ACKNOWLEDGMENTS}

The work is supported by IPA (Information Promotion Agency), Ministry of International Trade and Industry, Japan. We would like to thank Brent N. Reeves, Kaoru Rin Hayashi, Kouichi Kishida, Akira Kumagai, members of the CCC Lab at NAIST, and members of the L3D Center at University of Colorado at Boulder, for their valuable contributions to the project. We would also like to thank people who participated in our case studies.

\section{REFERENCES}

Aoki, A., Nakakoji, K., Suzuki, H., Mizushima, K., (1995) Experience and Analysis of ImageSearcher in the Smalltalk Environment: an Image Retrieval System by Analyzing Properties of Images, Proceedings of the Object-Oriented Symposium'95, IPSJ, Tokyo, Japan, June, pp. 253-260, (in Japanese).

Bearne, M., Jones, S., Sapsford-Francis, J., (1994) Towards Usability Gudelines for Multimedia Systems, Proceedings of ACM Multimedia'94, ACM Press, San Francisco, CA, October, pp. 105-110.

Boden, M. (1991) The Creative Mind: Myths \& Mechanisms, Basic Books.

Csikszentmihalyi, M., Sawyer, K., (1995) Creative Insight: The Social Dimension of a Solitary Moment,
The Nature of Insight (R.J. Sternberg, J.E. Davidson Eds.), MIT Press, Cambridge, MA, Chap. 11, pp. 329364.

Faraday, P. and Sutcliffe, A. (1997) Designing Effective Multimedia Presentations. Proceedings of CHI'97, ACM Press, Atlanta, GA, (forthcoming).

Fischer, G, Nakakoji, K, Ostwald, J, Stahl, G, Sumner, $T$ (1993) Embedding Computer-Based Critics in the Contexts of Design, Human Factors in Computing Systems, INTERCHI'93 Conference Proceedings (Amsterdam, Netherlands), ACM, pp. 157-164.

Fischer, G. and Nakakoji, K., (1994) Amplifying Designers' Creativity with Domain-Oriented Design Environments, Artificial Intelligence and Creativity ( $\mathrm{T}$. Dartnall ed.), Kluwer Academic Publishers, the Netherlands, pp.343-364.

McLaughlin, S, Gero, J.S., (1989) Creative Processes Can They Be Automated?, Modeling Creativity and Knowledge-Based Creative Design (Reprints of the International Round-Table Conference: Modeling Creativity and Knowledge-Based Creative Design), Heron Island, Queensland, December, pp. 69-94.

Nakakoji, K. Sumner, T, and Harstad, B (1994) Perspective-Based Critiquing: Helping Designers Cope with Conflicts Among Design Intentions, Artificial Intelligence in Design'94, J. Gero and F. Sudweeks (eds.), Kluwer Academic Publishers, Lausanne, Switzerland, August, pp. 449-466.

Nakakoji, K., Reeves, B.N., Aoki, A., Suzuki, H., Mizushima, K., (1995) eMMaC: Knowledge-Based Color Critiquing Support for Novice Multimedia Authors, Proceedings of ACM Multimedia'95, ACM Press, San Francisco, CA, pp. 467-476, November.

Nakakoji, K., Aoki, A., Reeves, B.N., KnowledgeBased Cognitive Support for Multimedia Information Design, Information and Software Technology, Elsevier Science, Vol. 38, No.3, pp.191-200, January. Sidelinger, S J (1985), Color Manual, Prentice-Hall, Inc., (Englewood Cliffs, NJ).

Terveen, L.G., An Overview of Human-Computer Collaboration, Knowledge-Based Systems Journal, Vol. 8, No. 2-3, April-June 1995, pp. 67-81.

Tufte, E.R., (1983) The Visual Display of Quantitative Information, Graphics Press, Cheshire, CT. 